\title{
DEVELOPMENT OF ANN-BASED MODELS TO PREDICT THE BOND STRENGTH OF GFRP BARS AND CONCRETE BEAMS
}

\author{
Thuy-Anh Nguyen*, Hai-Bang Ly \\ University of Transport Technology, 54 Trieu Khuc, Thanh Xuan, Hanoi, Vietnam \\ ARTICLE INFO
}

TYPE: Research Article

Received: 5/5/2020

Revised: $10 / 9 / 2020$

Accepted: 22/9/2020

Published online: 30/9/2020

https://doi.org/10.47869/tcsj.71.7.7

* Corresponding author

Email: anhnt@utt.edu.vn; Tel: 0988087009

\begin{abstract}
The use of glass fiber-reinforced polymer (GFRP) has gained increasing attention over the past decades, aiming at replacing traditional steel rebar in concrete structures, especially in corrosion or magnetic conditions. Understanding the working mechanism between the reinforcements and concrete is crucial in many practical applications, in which the corresponding bond strength is considered as a critical element. In this study, a database including 159 experimental beam results gathered from the available literature was used for the development of an artificial neural network (ANN) model in an effort to predict the bond strength between GFRP bars and concrete. Two ANN models using BFGS quasi-Newton backpropagation and conjugate gradient backpropagation with Polak-Ribiére algorithms were constructed and evaluated in terms of bond strength prediction accuracy. The considered database consisted of five input parameters, including the bar diameter, concrete compressive strength, minimum cover to bar diameter ratio, bar development length to bar diameter ratio, the ratio of the area of transverse reinforcement to the product of transverse reinforcement spacing, the number of developed bars and bar diameter. The evaluation of the models was conducted and compared using well-known statistical measurements, namely the correlation coefficient (R), root mean square error (RMSE), and absolute mean error (MAE). The results demonstrated that both ANN models could accurately predict the bond strength between GFRP bars and concrete, paving the way for engineers to possess a useful alternative design solution for reinforced concrete structures.
\end{abstract}

Keywords: bond strength, GFRP bars, Artificial Intelligence (AI), Artificial Neural Network (ANN). 


\section{INTRODUCTION}

Reinforced concrete remains the primer composition used in the construction fields over the decades. Generally, reinforcing rods are used in traditional reinforced concrete structures to bear the entire tensile stresses generated, under loading conditions and over the entire service life. Despite the outstanding advantages thanks to the reasonable combination of concrete and reinforced components, rust or corrosion of the reinforcement bars is the main disadvantage of this material. Besides, reinforced concrete does not possess conductive properties for lightning protection for buildings or passing of electromagnetic waves. In order to overcome the above disadvantages, recent developments have successfully applied a new type of reinforcement, the so-called glass fiber-reinforced polymer (GFRP) [1]. Indeed, GFRP is environmentally friendly and can replace the rebars under specific conditions or not suitable for a conventional type of reinforcement, such as coastal buildings. GFRP is a composite technology product, in which glass fiber is the main bearing part and accounting for $75 \%$ weight. The fiberglass bundles are bound and bound together by a binder of an epoxy mixture, hardener, and denatured component, which then runs through the heating furnace to form polymer steel [2]. GFRP bars are recognized as a superior alternative to ordinary steel bars, mainly for their high strength, lightweight, noncorrosive, fatigue resistance, nonmagnetic electrical insulation, and small creep deformation. The use of GFRP bars could prevent deterioration due to corrosion, improve durability, and increase the service life of structures [3]. On the basis of the mechanical properties, the bond strength between GFRP bars and concrete is one of the important factors in reinforced concrete structures. The characteristic of GFRP material is anisotropic, non-homogeneous, and linear elastic, thus resulting in different force transfer mechanisms between reinforcements and concrete [4]. Accordingly, accurate prediction of the bond strength between the GFRP bars and concrete is crucial for the design of load-bearing structures.

Over the past few decades, many studies have been performed to identify the main factors affecting the bonding behavior based on either beam or direct pullout tests [5]-[7]. These empirical studies have shown some main parameters affecting the bond strength, such as concrete compressive strength, concrete cover, bar diameter, bar position, bar surface, and development length. Besides, theoretical studies have also been carried out to analyze the bond mechanism of GFRP with concrete. Based on the experimental results, three theoretical bond-slip relationships, namely the Malvar model [8], the Bertero-Popov-Eligehausen model [9], and the Cosenza-Manfredi-Realfonzo (CMR) model [10], were introduced to evaluate the adequacy to model FRP-concrete bond. Three most promising models for GFRP rebars were evaluated and compared with a finite element model for the analysis of GFRP-reinforced concrete beams. Besides, several works used these models to analyze the bond behavior of GFRP bars in different materials and environments [11], [12]. Aside from, the design codes of the United States (United States) [13], Canada [14], and Japan [15] for GFRP bars also provided guidelines related to the bond mechanism in terms of both development length and 
bond strength. However, in reality, no uniform manufacturing standards have been established for GFRP bars, leading to the difference of FPR bars performance in different countries and manufacturers. Therefore, existing models proposed to analyze the bond stress-slip relations have made certain assumptions [16]. So far, no unified model has been universally applicable to the bond behavior of the GFRP bar. Therefore, it is necessary to conduct researches on a universal method to predict the bond strength between GFRP bars and concrete.

Over the past decades, machine learning algorithms have been successfully applied in the field of civil engineering, especially Artificial Neural Network (ANN) [17] - [19]. The ANN is a mathematical model built on the understanding of information processing processes of the human brain. So far, thanks to the advances in theory, calculation ability, and practical advantages, the ANN model has been increasingly applied in many different technical fields, especially in applications complex function simulations to find the relationship between input and output variables. In a study by Dahou et al. [20], an ANN-based model was used to predict the final bond strength of ribbed reinforcement bars, derived from pull-out tests. In another work of Golafshani et al. [21], an ANN and fuzzy logic (FL) models were developed to predict the bond strength of steel bars in concrete. The predictive results show that it is acceptable for both ANN and FL models, in which ANN models exhibited higher accuracy than FL. This fact clearly highlighted the applicability of the ANN model to solve complex technical problems. However, limited studies have investigated the robustness of the ANN algorithm and shown only the results corresponded to the best predictor.

Therefore, in this study, two ANN models using BFGS quasi-Newton backpropagation and Conjugate gradient backpropagation with Polak-Ribiére algorithms are developed. Moreover, the robustness of the two models are evaluated using mean and standard deviation values of three criteria to fully assess the robustness of the algorithms in predicting the bond strength of GFRP bars in concrete.

\section{METHODS USED}

\subsection{Artificial neural network}

Artificial neural network (ANN) is a model of information processing simulated based on the activity of the biological nervous system, consisting of a large number of neurons that are attached to process information. ANN has the ability to store information and use such information in the prediction of unknown data. The general architecture of an ANN consists of 3 components, which are the input layer, the hidden layer, and the output layer. The relationship between the input data and the result is shown by equation (1)

$$
Y=f\left(X_{1}, X_{2}, \ldots, X_{n}\right)
$$

in which $Y$ is the result to be forecasted, and $X_{1}, X_{2}, \ldots, X_{n}$ are the number of input data vectors.

These data classes are linked through weights, bias, and transformation functions. 


\subsection{Training algorithms}

Network training is the process of determining the optimal weight of an ANN. This is done by determining the performance function (usually the average square error between the network output and the desired target) and then minimizing it by weights. The backpropagation (BP) algorithm is the most widely used training technique for continuous function mapping, which has proven to be theoretically correct. The BP algorithm consists of two stages. The first is the signal propagation phase (Signal Forward). The input signals (vector of input values) are propagated from the input layer to the output layer (passing through hidden layers). The next stage is the backward error propagation (Error Backward). Based on the desired output value of the input vector, the system will calculate the error value. The error value is propagated backward from the output layer to the input layer. The weights will be updated (or adjusted) until the error is considered acceptable. The weight adjustment is made according to the generalized delta rule to minimize the error [22]. However, BPNN generally converges slowly and could easily be trapped in a local minimum. In order to avoid these disadvantages, various training algorithms have been proposed to speed up the ANN training phase. In this study, two different weight update functions were evaluated, namely the Broyden-Fletcher-Goldfarb-Shanno (BFGS) Quasi-Newton backpropagation (simply denoted as BFG) and conjugate gradient backpropagation with Polak-Ribiére updates (CGP) to optimize the predicted bond strength of GFRP bar in concrete.

\subsubsection{BFGS quasi-Newton backpropagation (BGF)}

The BFGS method belongs to quasi-Newton methods, a class of hill-climbing optimization techniques that seek a stationary point of a (preferably twice continuously differentiable) function. In Quasi-Newton methods, the Hessian matrix of second derivatives is not computed. Instead, the Hessian matrix is approximated using updates specified by gradient evaluations (or approximate gradient evaluations). The BFGS method is one of the most popular members of this class. This algorithm approximates the Hessian matrix by a function of the gradient to reduce the computational and storage requirements. The update of the approximation to the Hessian matrix on iteration $r$ can be written as:

$$
\Delta B^{r}=\frac{\left(\nabla E\left(\omega^{r}\right)-\nabla E\left(\omega^{r-1}\right)\right)\left(\nabla E\left(\omega^{r}\right)-\nabla E\left(\omega^{r-1}\right)\right)^{T}}{\left(\nabla E\left(\omega^{r}\right)-\nabla E\left(\omega^{r-1}\right)\right)^{T} \Delta \omega^{r-1}}-\frac{B^{r-1} \Delta \omega^{r-1}\left(B^{r-1} \Delta \omega^{r-1}\right)^{T}}{\left(\Delta \omega^{r-1}\right)^{T} B^{r-1} \Delta \omega^{r-1}}
$$

where $\Delta B^{r}$ refers to the update of the approximation to the Hessian matrix on iteration $r$, and $B^{r-1}$ is the approximation to the Hessian matrix on iteration $r-1$.

\subsubsection{Conjugate gradient backpropagation with Polak-Ribiére (CGP)}

The basic BP algorithm adjusts the weights in the steepest downward direction. In conjugate gradient algorithms, the first iteration is started by searching in the steepest downward direction (negative of the slope):

$$
p_{0}=-g_{0}
$$

A search is then conducted along the conjugate gradient direction to determine the optimal distance to minimize the performance function along that line. The conjugate gradient 
used here is proposed by Polak and Ribiére [23]. The search direction at each iteration is determined by updating the weight vector as:

$$
\begin{gathered}
\omega_{k+1}=\omega_{k}+\alpha_{k} p_{k} \\
p_{k}=-g_{k}+\beta_{k} p_{k-1} \\
\beta_{k}=\frac{\Delta g_{k-1}^{T} g_{k}}{g_{k-1}^{T} g_{k-1}} \\
\Delta g_{k-1}^{T}=g_{k}^{T}-g_{k-1}^{T}
\end{gathered}
$$

where:

\subsection{Performance criteria}

In order to validate developed ANN models, three statistical criteria, namely correlation coefficient (R), root mean square error (RMSE), and absolute mean error (MAE) were used in this research. The $\mathrm{R}$ value used to investigate the linear correlation between the target and the predicted values, lies in the range $[-1 ; 1]$. Both RMSE and MAE measure the average intensity of the error, where MAE represents the residual error between the target and predicted values for each dataset, and RMSE denotes the square root of the average residual error between target values and predicted values for each data set. For these two indicators, the smaller value signifies the better performance of the ANN model. The closer the absolute value of $\mathrm{R}$ is to 1 , the stronger the ANN model is in predicting bond strength. R, RMSE, and MAE are defined by the following equations:

$$
\begin{gathered}
R M S E=\sqrt{\frac{1}{N} \sum_{j=1}^{N}\left(p_{0, j}-p_{t, j}\right)} \\
M A E=\frac{1}{N} \sum_{j=1}^{N}\left|p_{0, j}-p_{t, j}\right| \\
R=\sqrt{\frac{\sum_{j=1}^{N}\left(p_{0, j}-\bar{p}_{0}\right)\left(p_{\mathrm{t}, j}-\bar{p}_{t}\right)}{\sqrt{\sum_{j=1}^{N}\left(p_{0, j}-\bar{p}_{0}\right)^{2} \sum_{j=1}^{N}\left(p_{\mathrm{t}, j}-\bar{p}_{t}\right)^{2}}}}
\end{gathered}
$$

where: $N$ is the number of database, $p_{0}$ and $\bar{p}_{0}$ is the actual experimental value and the average real experimental value, $p_{t}$ and $\bar{p}_{t}$ is the predicted value and the average predicted value, calculated according to the model forecast.

\section{CONSTRUCTION OF DATABASE}

The development database for this study, including 159 beam test data is published in the literature [24]. The set of tested beam-type specimens consisting of notched, hinged, splice, and inverted hinged beam specimens. ANN model built using five input parameters, the bar diameter $\left(\mathrm{d}_{\mathrm{b}}\right)$, concrete compressive strength $\left(\mathrm{f}_{\mathrm{c}} \mathrm{c}\right)$, minimum cover to bar diameter ratio $\left(\mathrm{C} / \mathrm{d}_{\mathrm{b}}\right)$, bar development length to bar diameter ratio $\left(1 / \mathrm{d}_{\mathrm{b}}\right)$ and the ratio of the area of transverse reinforcement to the product of transverse reinforcement spacing, the number of developed bars and bar diameter $\left(\mathrm{A}_{\mathrm{tr}} / \mathrm{snd}_{\mathrm{b}}\right)$. The output parameter is the bond strength. In the 
collected dataset, the value of the bar diameter varies in the range of 9.53-28.58 $\mathrm{mm}$, the concrete compressive strength is in the range $23.43-48.86 \mathrm{MPa}$, the liquid limit varies from 20.8-154.12\%, the minimum cover to bar diameter ratio ranges between 1 and 6.2 , the bar development length to bar diameter ratio value varies from 3.56 to 97.24 , and the number of developed bars and bar diameter ranges from $0-0.08 \mathrm{~mm}$. Besides, the bond strength values are in the range of 1.64 to $22.34 \mathrm{MPa}$. The detailed statistical information of these variables is shown in Table 1. An illustrative schematic of the beam test is shown in figure 1.

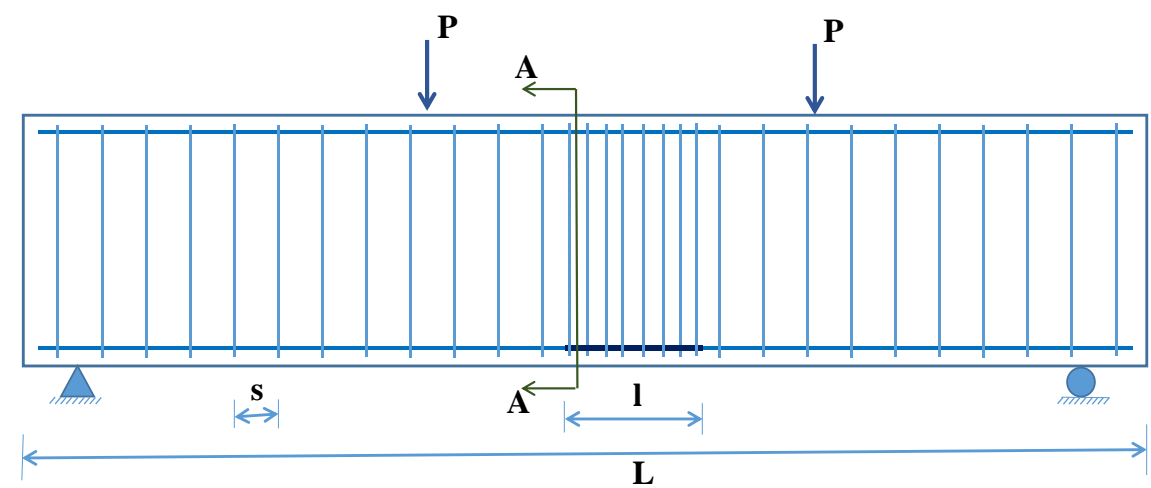

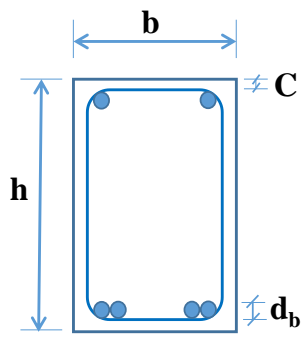

$\underline{\text { Section A-A }}$

Figure 1. Schematic illustration of beam test.

The dataset was randomly divided into two subsets using a uniform distribution, in which $70 \%$ of the data (corresponding to 111 data) was used to train ANN models, and $30 \%$ of the remaining data (corresponding to 48 data) was used for model verification. All data are scaled within the range of $[0,1]$ to reduce number errors while processing by ANN, according to the recommendations of. This process ensures that the training phase of AI models can be carried out with functional generalization capabilities. Such proportions are expressed using equation (10):

$$
\chi_{\text {scaled }}=\frac{2(\chi-\lambda)}{\mu-\chi}-1
$$

where $\lambda$ and $\mu$ are the minimum and maximum values of given variables, and $\chi$ is the value of the variable to be scaled.

Table 1. Statistical analysis of the input and output variables used in this study.

\begin{tabular}{|l|c|c|c|c|c|c|c|}
\hline & Unit & Role & Min & Median & Average & Max & StD* \\
\hline $\mathrm{d}_{\mathrm{b}}$ & $\mathrm{Mm}$ & Input & 9.530 & 18.440 & 18.675 & 28.580 & 5.033 \\
\hline $\mathrm{f}_{\mathrm{c}}$ & $\mathrm{MPa}$ & Input & 23.430 & 31.020 & 34.972 & 48.860 & 7.109 \\
\hline $\mathrm{C} / \mathrm{d}_{\mathrm{b}}$ & - & Input & 1.000 & 2.120 & 2.661 & 6.200 & 1.153 \\
\hline $1 / \mathrm{d}_{\mathrm{b}}$ & - & Input & 3.560 & 16.000 & 18.161 & 97.240 & 15.813 \\
\hline $\mathrm{A}_{\mathrm{tr}} / \mathrm{snd}_{\mathrm{b}}$ & $\mathrm{mm}$ & Input & 0.000 & 0.000 & 0.016 & 0.080 & 0.023 \\
\hline$\tau_{\mathrm{b}}$ & $\mathrm{MPa}$ & Output & 1.640 & 5.880 & 7.829 & 22.340 & 5.042 \\
\hline
\end{tabular}

StD $*$ standard deviation 


\section{RESULTS AND DISCUSSION}

For ANN model training process and backpropagation network structure, the critical internal parameters include data preprocessing and presentation, initial synaptic weights, learning rate, number of hidden layers and number of neurons in each hidden layer, activation functions for hidden layers and output layers, and the number of training epochs. The number of hidden layers is usually determined during the ANN network structure setup. The number of hidden layers needed for the network depends on the complexity of the relationship between the input parameters and the output value. Currently, researchers have not yet come up with a method to determine the optimal structure of the ANN network. The process of building a network structure is a process of trial and error. Many network models are given by changing the number of neural in each hidden layer and the number of hidden layers in the model. The model gives the best forecast results will be chosen. Cybenko [25] and Bound [26] succeeded in using a hidden layer model in classifying input variables to process models. Determining the number of hidden layers, starting with a hidden layer, then increasing the number of neural in that hidden layer. If the model accuracy is still not good, the final choice is to increase the number of hidden layers, according to the author. The amount of neural in each hidden layer depends on the problem to be solved. In this study, the ANN model structure for predicting the bond strength of GFRP bars in concrete was selected with three layers, in which: the input layer consists of 5 neurons corresponding to 5 input parameters, the hidden layer consists of 9 neurons, and the output layer included one neuron representing the bond strength.

\subsection{BGF-ANN and CGP-ANN network performance}

ANN-BFG model and ANN-CGP model with structure (5-9-1) were used in this study to predict the bond strength of GFRP bars in concrete. As mentioned above, the performance of ANN model depends on the number of training epochs. The trial and error process is used to determine the optimal number of training epochs. Figure 2 shows the best training performance for the training dataset of the ANN-BFG model (Figure 2a) and ANN-CGP model (Figure 2b), while Figure $2 \mathrm{c}$ and Figure $2 \mathrm{~d}$ represent some examples while the models were overfitting if the number of epochs was not correctly taken. As observed, the ANN-BFG model for the training phase with 50 epochs was optimal, whereas the ANN-CGP model exhibited the best performance with 100 epochs. These numbers of epochs were selected to avoid overfitting, where the network has not removed the noise itself, so the output destination function is only true for the dataset put into network training, and other data will bring substantial errors. 
Transport and Communications Science Journal, Vol. 71, Issue 7 (09/2020), 814-827

(a)

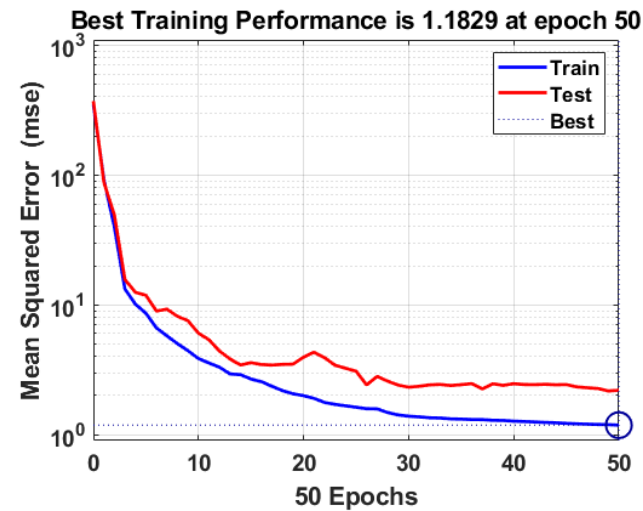

(c)

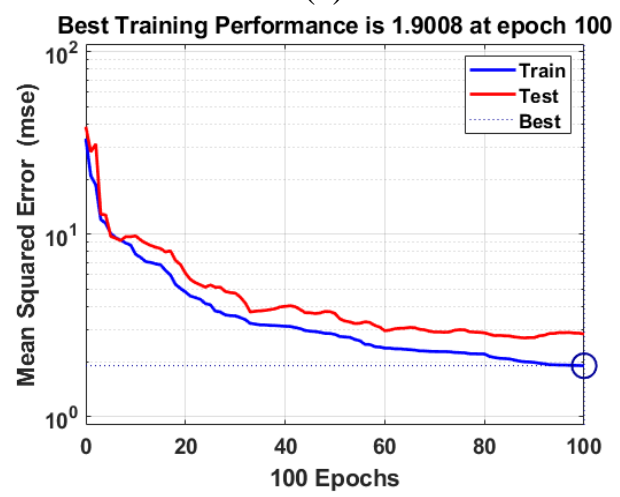

(b)

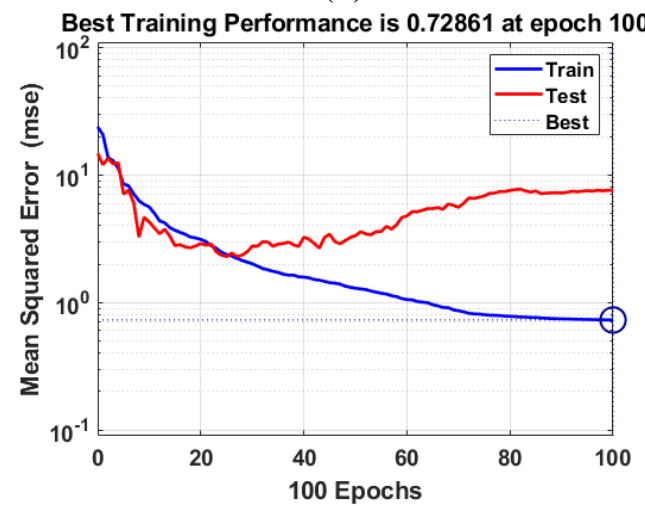

(d)

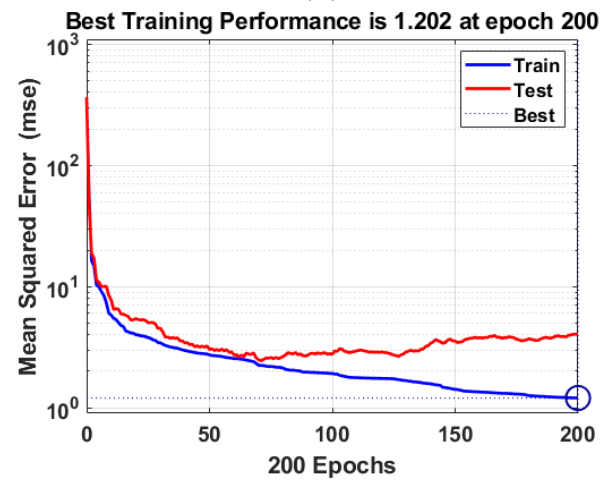

Figure 2. A graph showing the best training performance of (a) ANN-BFG, (b) ANN-CGP, and the potential error in using the wrong number of epochs (c) ANN-BFG; and (d) ANN-CGP.

\subsection{Convergence of results}
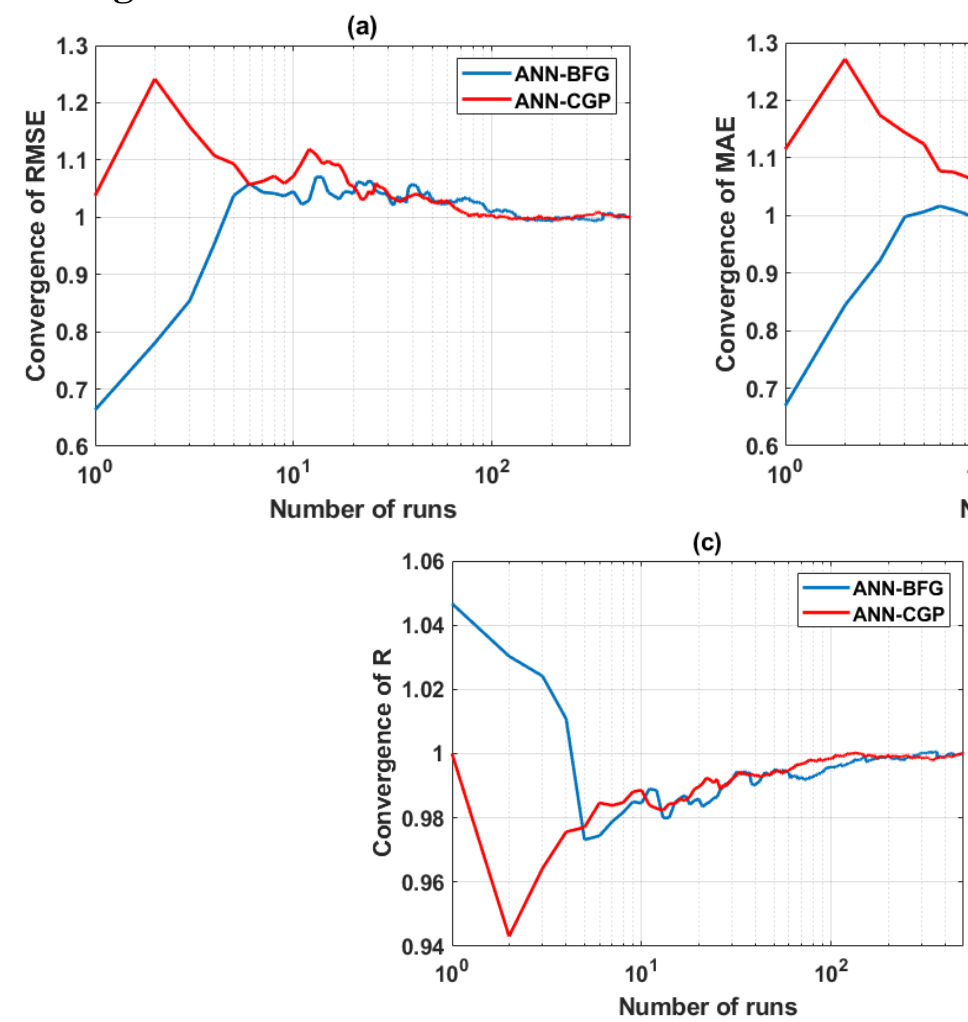

Figure 3. Normalized statistical convergence over 500 simulations for: (a) RMSE; (b) MAE; and (c) R. (b)

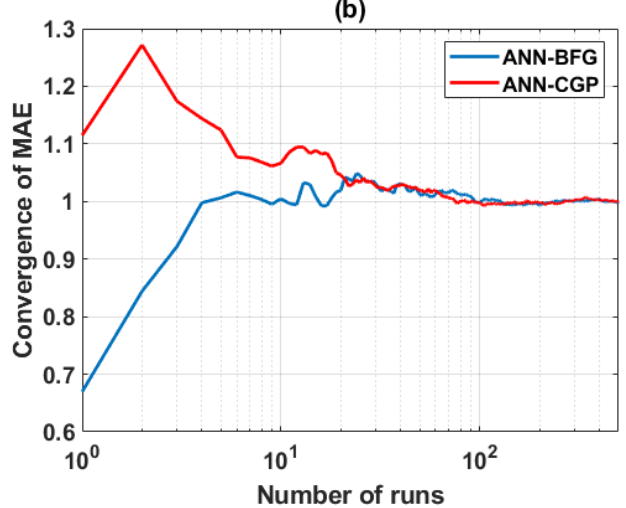


The convergence of the proposed ANN model depends on the number of simulations for each ANN combination. In this study, a number of 500 simulations were employed to study the performance of the proposed ANN models in the presence of input variability. The predicted convergence concerning RMSE, MAE, and R for the ANN-CGP model (red line) and ANN-BFG model (green line) is plotted in Figure 3. As observed, after about 70 simulations, the fluctuations of RMSE and MAE are in the 5\% range, and below $1 \%$ for R. When the number of simulations reached 500, all values of RMSE, MAE and R are converged. It is shown that the choice of 500 simulations was relevant in order to obtain optimized results for all R, RMSE, and MAE values. The prediction capability of each ANN model is presented in the next section.

\subsection{Prediction capability}
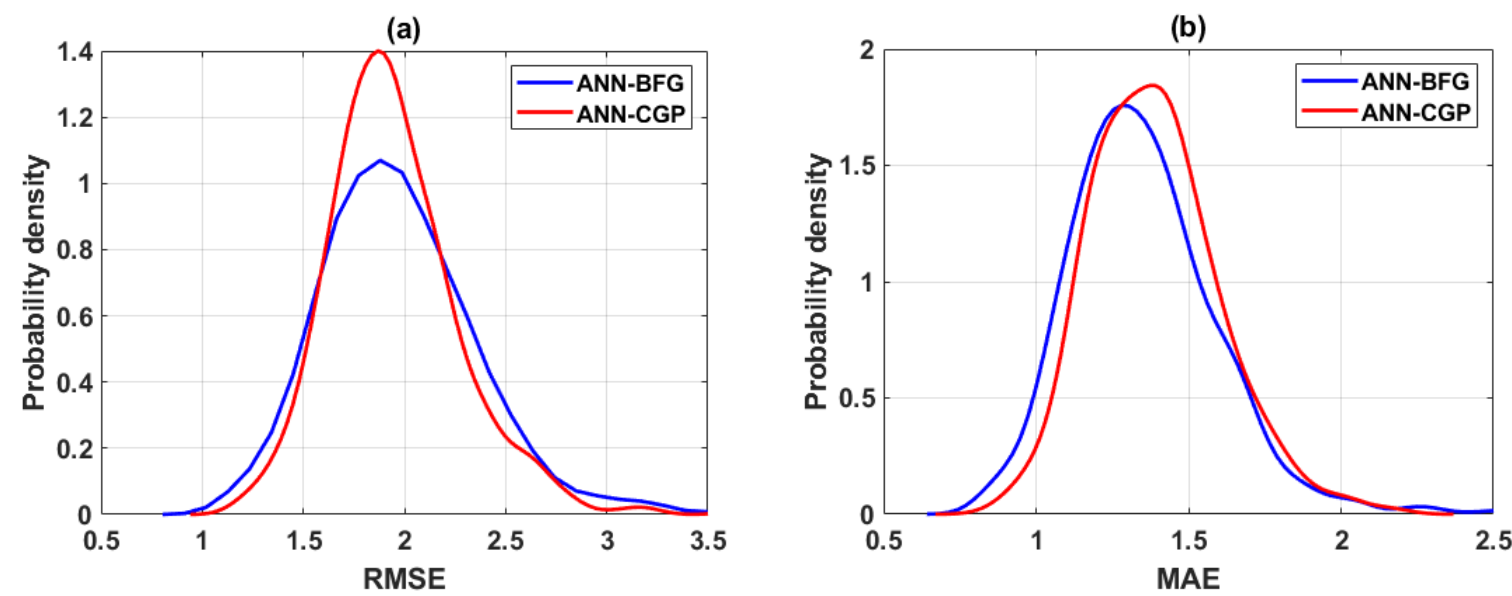

(c)

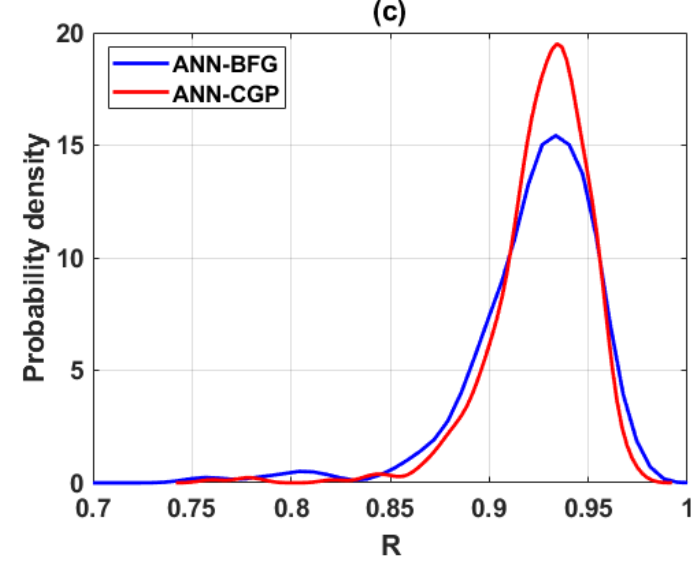

Figure 4. Probability density function of (a) RMSE, (b) MAE, and (c) R over 500 simulations using ANN-BFG and ANN-CGP, respectively.

The probability density function of RMSE, MAE, and R values for the ANN-BFG model and ANN-CGP model is shown in Figure 4. The statistical analysis results shown in Table 2 include the min, mean, max, median, and standard deviation (St.D) of variation of R, RMSE, and MAE distributions. The mean and standard deviation values corresponding to the case of $\mathrm{R}$ were $0.921,0.0456$ for ANN-BFG, and 0.9265, 0.0252 for ANN-CGP, respectively. With 
Transport and Communications Science Journal, Vol. 71, Issue 7 (09/2020), 814-827

respect to RMSE, these values were 2.0281, 0.6840 for ANN-BFG, and 1.9455, 0.3225 for ANN-CGP, respectively. The values of average and standard deviation of MAE were 1.3602, 0.2631 for ANN-BFG, and 1.3895, 0.2103 for ANN-CGP, respectively. From the statistical analysis of the obtained results, it is seen that the error distribution of the ANN-BFG model and ANN-CGP model were quite close to each other. However, the ANN-CGP model is slightly better ( $\mathrm{R}$ values was about $0.6 \%$ higher than the ANN-BFG model). This showed that both ANN-CGP and ANN-BGF produce excellent results in terms of bond strength prediction. Thus, the ANN models with backpropagation algorithms were promising to predict the bond strength of GFRP bars in concrete.

Table 2. Statistical analysis results of R, RMSE and MAE distributions over 500 simulations.

\begin{tabular}{lccccc}
\hline & Min. & Mean & Max & Median & St.D. \\
\hline RMSE(ANN-BFG) & 1.1346 & 2.0281 & 11.1313 & 1.9443 & 0.6840 \\
\hline MAE(ANN-BFG) & 0.8399 & 1.3602 & 2.9692 & 1.3231 & 0.2631 \\
\hline R(ANN-BFG) & 0.3512 & 0.9210 & 0.9778 & 0.9291 & 0.0456 \\
\hline RMSE(ANN-CGP) & 1.1979 & 1.9455 & 3.6454 & 1.9083 & 0.3225 \\
\hline MAE(ANN-CGP) & 0.8628 & 1.3895 & 2.1757 & 1.3759 & 0.2103 \\
\hline R(ANN-CGP) & 0.7597 & 0.9265 & 0.9744 & 0.9300 & 0.0252 \\
\hline \hline
\end{tabular}

\subsection{Typical prediction results}

In this section, the validation of two ANN models is proposed to predict the bond strength of glass fiber-reinforced polymer concrete. The regression graph between the actual and predicted results for the training dataset is shown in Figures 5a and 5c, whereas Figures $5 \mathrm{~b}$ and $5 \mathrm{~d}$ show the correlation for the testing dataset, using ANN-BGF and ANN-CGP, respectively. A linear fit was also applied and plotted in each case. The values of $\mathrm{R}$ calculated for the training dataset were 0.97016 for ANN-BFG model, and 0.95461 for ANN-CGP model. For the testing dataset, these values were $0.97416,0.97438$ for ANN-BFG, and ANNCGP models, respectively.

The results obtained in this study were found more accurate than previously published results in the literature. For instance, in the work of Golafshani et al. [24], the authors proposed an ANN model and obtained the RMSE values of 1.27 and 1.37, and MAE values of 
0.97 and 1.06 for the validating and testing datasets, respectively. In another work by Yan et al. [27], the MAE values were 0.85 and 0.84 , whereas those of RMSE were 1.35 and 1.31 for the validating and testing parts, respectively. Besides, Yan et al. [28] obtained the R values using ANN with genetic optimization (GA) of 0.948 and 0.951 for the validation and testing parts, respectively. These results were inferior to those of the present work, considering the best performance of $\mathrm{ANN}-\mathrm{BFG}$ herein: $\mathrm{RMSE}=1.135, \mathrm{MAE}=0.840$, and $\mathrm{R}=0.9778$ (see Table 2).

(a)

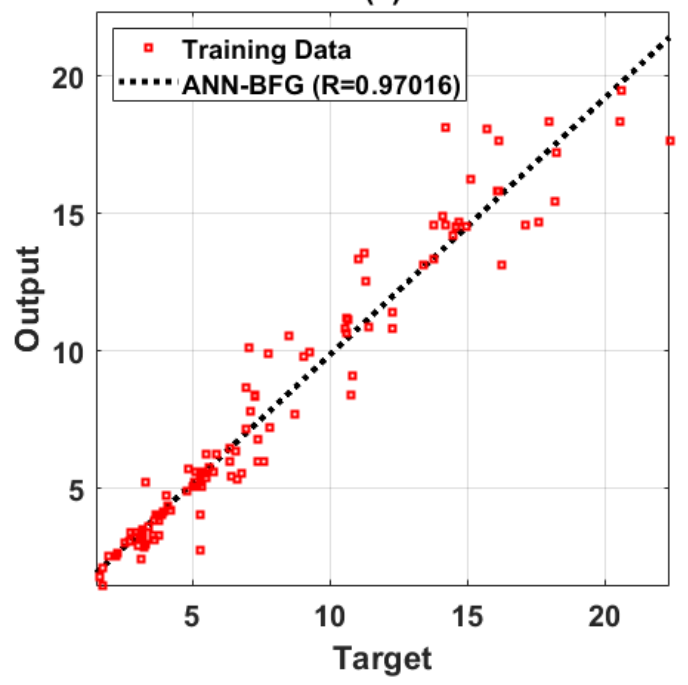

(c)

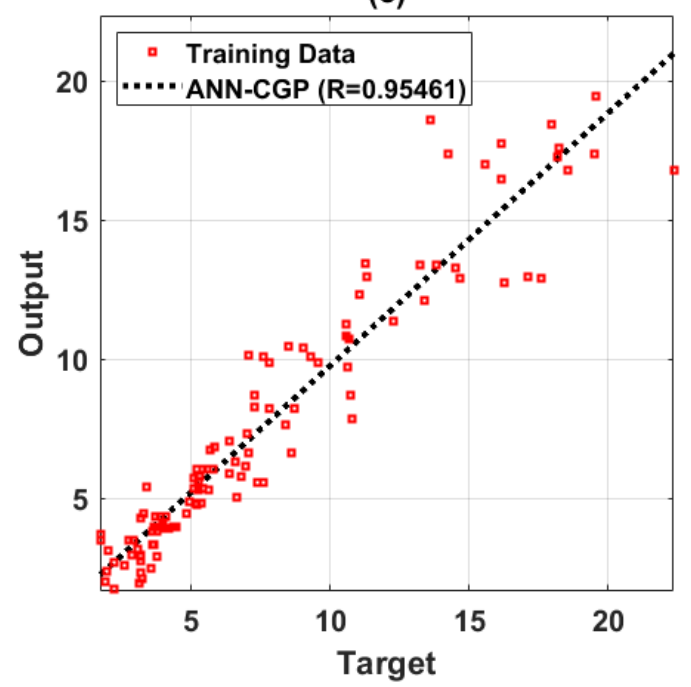

(b)

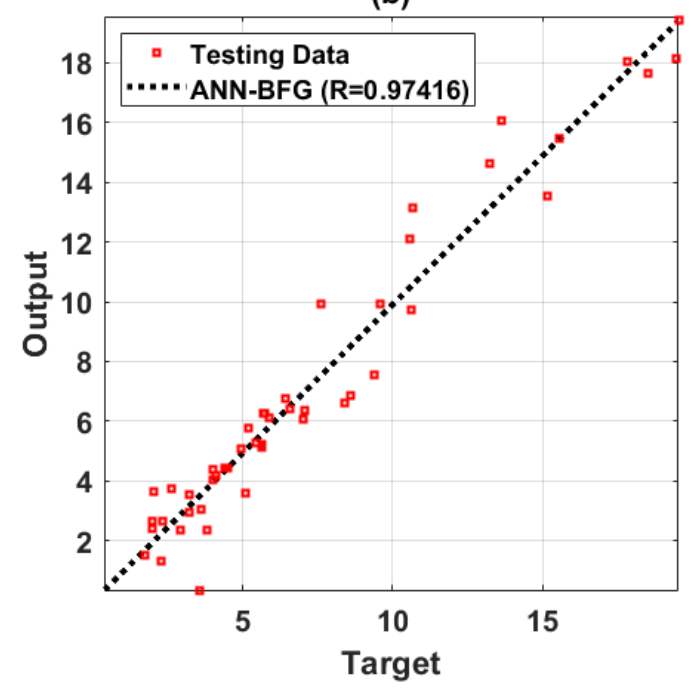

(d)

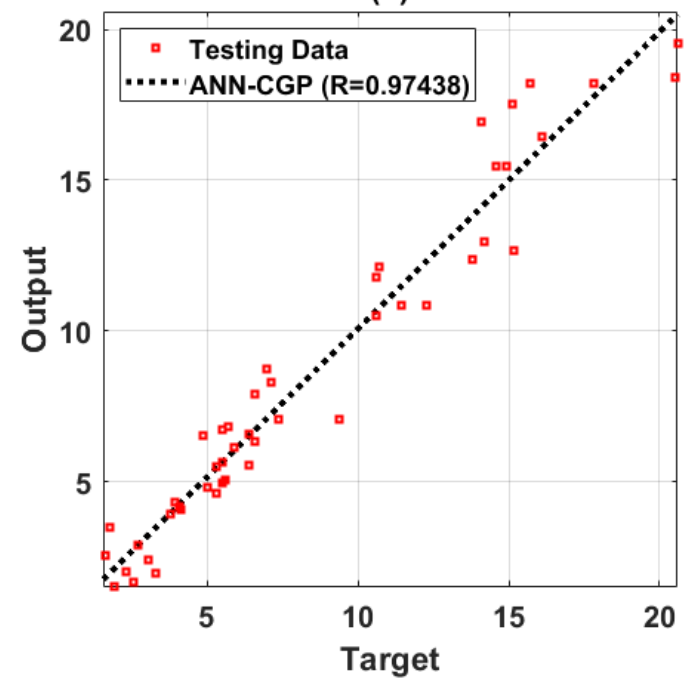

Figure 5. Regression graphs between predicted and actual values of the bond strength of GFRP bar in concrete for the training dataset using: (a) ANN-BFG, (c) ANN-CGP; for the testing dataset using: (b) ANN-BFG, (d) ANN-CGP, respectively. 


\section{CONCLUSION}

The ANN-BFG model and the ANN-CGP model developed in this study were used to predict the bond strength of GFRP bars in concrete. For this purpose, a database consisting of 159 different experimental bond beam tests was compiled, of which 111 records were randomly used for the training phase, and 48 were used for the testing phase. The analysis results showed that both models built were able to predict the bond strength of GFRP bars in concrete correctly, avoiding costly and difficult experimental tests that require detailed and specific training, equipment, expertise. The proposed simple ANN model can easily estimate the bonding strength of FRP bars in concrete so that structural engineers could predict the bond strength of GFRP bars in concrete.

\section{REFERENCES}

[1]. L. C. Bank, T. I. Campbell, C. W. Dolan, Guide for the Design and Construction of Concrete Reinforced with FRP Bars, Reported by ACI Committee 440, Concrete, 2003, pp. 1-42. https://www.iranfrp.ir/wp-content/uploads/2018/12/13.pdf

[2]. J. Rovira, A. Almerich, J. Molines, P. Martin, Develpoment and applications of glass fiber bars as a reinforced in concrete structures, 2011, pp. 1-6. https://www.researchgate.net/publication/289730640_Develpoment_and_applications_of_glass_fiber_ bars as a reinforced in concrete structures

[3]. K. M. A. Hossain, Bond Strength of GFRP Bars Embedded in Engineered Cementitious Composite using RILEM Beam Testing, International Journal of Concrete Structures and Materials, 12 (2018). https://doi.org/10.1186/s40069-018-0240-0

[4]. F. Yan, Z. Lin, M. Yang, Bond mechanism and bond strength of GFRP bars to concrete: A review, Compos. Part B Eng., 98 (2016) 56-69. https://doi.org/10.1016/j.compositesb.2016.04.068

[5]. B. T. B. Benmokrane, 0. Chaallal, Bond Strength and Load Distribution of Composite GFRP Reinforcing Bars in Concrete, ACI Mater. J., 93 (1996) 254-259. https://doi.org/10.14359/9810

[6]. B. Tighiouart, B. Benmokrane, D. Gao, Investigation of bond in concrete member with fibre reinforced polymer (FRP) bars, Constr. Build. Mater., 12 (1998) 453-462. https://doi.org/10.1016/S0950-0618(98)00027-0

[7]. H. Mazaheripour, J. Barros, J. Sena-Cruz, M. Pepe, E. Martinelli, Experimental study on bond performance of GFRP bars in self-compacting steel fiber reinforced concrete, Compos. Struct., 95 (2013) 202-212. https://doi.org/10.1016/j.compstruct.2012.07.009

[8]. L. J. Malvar, Bond Stress-Slip Characteristics of FRP Rebars, (No. NFESC-TR-2013-SHR), Nav. Facil. Eng. Serv. Cent. Port Hueneme, CA, USA, 1994.

[9]. V. Eligehausen, R., Popov, E.P., Bertero, Local Bond Stress-Slip Relationships of Deformed Bars under Generalized Excitations, Rep. No. 83/23; Earthq. Eng. Serv. Center, Univ. Calif. Berkeley, CA, USA, 1983.

[10].R. Cosenza, E., Manfredi, G., Realfonzo, Analytical modelling of bond between frp reinforcing bars and concrete, Non-Metallic Reinf. Concr. Struct. Proc. Second Int. RILEM Symp. RC Press London, $\quad$ England, $29 \quad$ (1995) 164-171. https://www.researchgate.net/publication/262259677_Analytical_modelling_of_bond_between_FRP_r einforcing_bars_and_concrete 
Transport and Communications Science Journal, Vol. 71, Issue 7 (09/2020), 814-827

[11].M. Pepe, H. Mazaheripour, J. Barros, J. Sena-Cruz, E. Martinelli, Numerical calibration of bond law for GFRP bars embedded in steel fibre-reinforced self-compacting concrete, Compos. Part B Eng., 50 (2013) 403-412. https://doi.org/10.1016/j.compositesb.2013.03.006

[12].W. J. Long, K. H. Khayat, G. Lemieux, S. D. Hwang, F. Xing, Pull-out strength and bond behavior of prestressing strands in prestressed self-consolidating concrete, Materials (Basel)., 7 (2014) 6930-6946. https://doi.org/10.3390/ma7106930

[13].ACI Committee 440.1R-06, Guide for the design and construction of concrete reinforced with FRP bars, Am. Concr. Inst., 2006, pp. 44.

[14].CAN, Design and Construction of Building Components with Fibre-Reinforced Polymers (CAN/CSA S806-02), Csa S806-02, no. Reaffirmed, 2009, pp. 177.

[15].A. Machida, T. Uomoto, Recommendation for design and construction of concrete structures using continuous fiber Recommendation for Design and Constr Continuous Fiber Rei Alsuhiko Machida Saitama University Takcto Uomoto University of Tokyo Summary This paper describes the outli, 1999.

[16].R. Masmoudi, A. Masmoudi, M. Ben Ouezdou, A. Daoud, Long-term bond performance of GFRP bars in concrete under temperature ranging from $20^{\circ} \mathrm{C}$ to $80^{\circ} \mathrm{C}$, Constr. Build. Mater., 25 (2011) 486-493. https://doi.org/10.1016/j.conbuildmat.2009.12.040

[17].H.B. Ly, T.A. Nguyen, Artificial neural network based modeling of the axial capacity of rectangular concrete filled steel tubes, Transport and Communications Science Journal, 71 (2020) 154-166. (in Vietnamese) https://doi.org/10.25073/tcsj.71.2.10

[18].H. Q. Nguyen, H. B. Ly, V. Q. Tran, T. A. Nguyen, T. T. Le, B. T. Pham, Optimization of artificial intelligence system by evolutionary algorithm for prediction of axial capacity of rectangular concrete filled steel tubes under compression, Materials (Basel)., 13 (2020). https://doi.org/10.3390/ma13051205

[19].H.-B. Ly et al., Prediction and Sensitivity Analysis of Bubble Dissolution Time in 3D Selective Laser Sintering Using Ensemble Decision Trees, Materials (Basel)., 12 (2019). https://doi.org/10.3390/ma12091544

[20].G. F. Dahou Z, Sbartai ZM, Castel A, Artificial neural network model for steel-concrete bond prediction, Eng. Struct., 31(2009) 1724-1733. https://doi.org/10.1016/j.engstruct.2009.02.010

[21].E. M. Golafshani, A. Rahai, M. H. Sebt, H. Akbarpour, Prediction of bond strength of spliced steel bars in concrete using artificial neural network and fuzzy logic, Constr. Build. Mater., 36 (2012) 411-418. https://doi.org/10.1016/j.conbuildmat.2012.04.046

[22].S. Haykin, "Neural Networks - A Comprehensive Foundation," 2nd Ed. Prentice Hall, 2000.

[23].R. S. E. Dennis, Numerical Methods for Unconstrained Optimization and Nonlinear Equations, Englewood Cliffs, NJ PrenticeHall, 1983.

[24].E. M. Golafshani, A. Rahai, M. H. Sebt, Artificial neural network and genetic programming for predicting the bond strength of GFRP bars in concrete, Mater. Struct., 48 (2015) 1581-1602. https://doi.org/10.1617/s11527-014-0256-0

[25].G. Cybenko, Approximation by superpositions of a sigmoidal function, Math. Control. Signals Syst., 2 (1989) 303-314. https://doi.org/10.1007/BF02551274

[26].Bounds, Lloyd, Mathew, Waddell, A multilayer perceptron network for the diagnosis of low back pain, in IEEE 1988 International Conference on Neural Networks, 2 (1988) 481-489. https://doi.org/10.1109/ICNN.1988.23963

[27].F. Yan, Z. Lin, X. Wang, F. Azarmi, K. Sobolev, Evaluation and prediction of bond strength of 
Transport and Communications Science Journal, Vol. 71, Issue 7 (09/2020), 814-827

GFRP-bar reinforced concrete using artificial neural network optimized with genetic algorithm, Compos. Struct., 161 (2017) 441-452. https://doi.org/10.1016/j.compstruct.2016.11.068

[28].F. Yan, Z. Lin, New strategy for anchorage reliability assessment of GFRP bars to concrete using hybrid artificial neural network with genetic algorithm, Compos. Part B Eng., 92 (2016) 420-433. https://doi.org/10.1016/j.compositesb.2016.02.008 